MATHEMATICS OF COMPUTATION

Volume 78, Number 268, October 2009, Pages 2371-2395

S 0025-5718(09)02233-9

Article electronically published on February 11, 2009

\title{
COMPUTATION OF HIGHLY RAMIFIED COVERINGS
}

\author{
RAIMUNDAS VIDŪNAS AND ALEXANDER V. KITAEV
}

\begin{abstract}
An almost Belyi covering is an algebraic covering of the projective line, such that all ramified points except one simple ramified point lie above a set of 3 points of the projective line. In general, there are 1-dimensional families of these coverings with a fixed ramification pattern. (That is, Hurwitz spaces for these coverings are curves.) In this paper, three almost Belyi coverings of degrees 11,12 , and 20 are explicitly constructed. We demonstrate how these coverings can be used for computation of several algebraic solutions of the sixth Painlevé equation.
\end{abstract}

\section{InTRODUCTION}

Recall that a Belyi function is a rational function on an algebraic curve with at most 3 critical values. The corresponding covering of $\mathbb{P}^{1}$ by the algebraic curve ramifies only above (at most) 3 points. By fractional-linear transformations, the ramification locus can be chosen to be the set $\{0,1, \infty\} \subset \mathbb{P}^{1}$.

According to Belyi [4] and Grothendieck [12], there are deep relations between Belyi functions and algebraic curves defined over $\overline{\mathbb{Q}}$, and dessins d'enfant.

More generally, one can consider the set of (isomorphism classes of) all coverings of $\mathbb{P}^{1}$ with a prescribed number of ramified points and with prescribed ramification orders above them. Such a topological configuration space is called a Hurwitz space. If we fix a ramification pattern for Belyi functions, we typically have a finite set of (isomorphism classes of) Belyi functions with the prescribed ramification pattern. If we fix the hypermap [33. of 3 permutations for the monodromy group of the covering, the Belyi map is unique.

In this article we consider coverings of $\mathbb{P}^{1}$ which ramify only above 4 points, and such that there is only one simple ramified point in one of the 4 fibers. We refer to these coverings as almost Belyi coverings. As is known, Hurwitz spaces for coverings ramified only above 4 general points have dimension one [33, Proposition 3.1]. In fact, any algebraic curve can be obtained as some one-dimensional Hurwitz space (with specified monodromy permutations) 9 .

For an almost Belyi covering of degree $n$, let us denote its ramification pattern by $R_{4}\left(P_{1}\left|P_{2}\right| P_{3}\right)$, where $P_{1}, P_{2}, P_{3}$ are the 3 partitions of $n$ specifying the ramification

Received by the editor June 21, 2007 and, in revised form, October 16, 2008.

2000 Mathematics Subject Classification. Primary 57M12, 34M55; Secondary 33E17.

Key words and phrases. Belyi map, dessin d'enfant, the Painlevé VI equation.

The first author was supported by the 21st Century COE Programme "Development of Dynamic Mathematics with High Functionality" of the Ministry of Education, Culture, Sports, Science and Technology of Japan.

The second author was supported by JSPS grant-in-aide No. 14204012. 
orders above three points. The fourth partition is assumed to be $2+1+1+\cdots+1$. The similar notation for a ramification pattern for Belyi maps is $R_{3}\left(P_{1}\left|P_{2}\right| P_{3}\right)$, as in [1, 19].

The main goal of this paper is to compute generic almost Belyi coverings $\mathbb{P}^{1} \rightarrow \mathbb{P}^{1}$ with the following ramification patterns:

$$
\begin{aligned}
& R_{4}(3+3+3+1+1+1|2+2+2+2+2+2| 5+5+2), \\
& R_{4}(3+3+3+1+1|2+2+2+2+2+1| 5+5+1), \\
& R_{4}(5+5+5+5|2+2+2+2+2+2+2+2+2+2| 3+3+3+3+3+2+1+1+1) .
\end{aligned}
$$

Their degrees are 12, 11 and 20, respectively.

We consider coverings with these ramification patterns because of their application to the theory of algebraic Painlevé VI functions. With certain almost Belyi coverings, one can pull back a hypergeometric differential equation to a parametric isomonodromic Fuchsian equation with 4 regular singular points plus one apparent singularity. Equivalently, one can obtain isomonodromic $2 \times 2$ matrix Fuchsian systems with 4 regular singular points. The corresponding Painlevé VI solutions are algebraic. Knowing suitable almost Belyi maps, one can construct explicit examples of algebraic Painlevé VI solutions ([18, [2], [10]), and solve the corresponding isomonodromic Fuchsian equations explicitly in terms of hypergeometric functions. More generally, explicit knowledge of any Hurwitz space can be similarly used to solve explicitly many types of Fuchsian systems, such as Garnier systems [17.

We construct three almost Belyi coverings, one for each ramification type in (1.1)-(1.3), and note that additionally there are two reducible coverings for the ramification pattern (1.3). With respect to the three interesting almost Belyi coverings, we pull back hypergeometric equations with the icosahedral monodromy group to isomonodromic $2 \times 2$ Fuchsian systems with 4 regular singular points and the same monodromy group. In total, we compute five corresponding algebraic Painlevé VI solutions in Section 6. They have types 37, 38, 41, 42, 43 in Boalch's classification of icosahedral Painlevé VI solutions. Three algebraic Painlevé VI solutions (of types $38,42,41$ ) can be constructed immediately 1 from the three almost Belyi coverings. To obtain the other two algebraic Painlevé VI solutions, we compute properly pull-backed corresponding Fuchsian systems explicitly. The type 41 solution is related to the Great Dodecahedron Solution of Dubrovin and Mazzocco [11] via an Okamoto transformation.

Efficient computations of highly ramified coverings or Hurwitz spaces are important problems in other fields as well. Therefore these problems attract the attention of researchers. In $[8$, a method is presented to compute one-dimensional Hurwitz spaces (for almost Belyi maps, for example) based on the degenerations when 4 ramification loci coalesce into 3 ramified points. In [22, a computer algebra package is presented for computing genera and monodromy groups of Hurwitz spaces or coverings.

\footnotetext{
${ }^{1}$ Our original motivation for this work was to compute a few missing examples in early versions of [5] of icosahedral Painlevé VI functions. We did our computations for type 38, 41 solutions unaware of the sixth electronic version of [5]. Before the next version of [5] in which type 42, 43 examples appeared, we had the degree 11 covering and the corresponding type 42 solution as well. Complementary to [5], computations of type 44-45 and 47-52 examples were done independently in [7] and 26].
} 
The paper is organized as follows. In the next section, we present our computational method. It is basically the same method as described in [30, Section 3]. Compared with the most straightforward method with undetermined coefficients, we derive equations of smaller degree in undetermined coefficients by using properties of the derivatives of Belyi or almost Belyi maps. We found out that very much the same computational method was used in [15], for deriving several rather simple Belyi coverings by hand. In Sections 3 through 5 we present our computations of the coverings with ramification patterns (1.1)-11.3). Section 6] demonstrates the mentioned application of our coverings to the computation of algebraic Painlevé VI solutions. The coverings are $R$-parts of $R S$-pullback transformations of hypergeometric differential equations to isomonodromic $2 \times 2$ Fuchsian systems. Computation of $R S$-pullback transformations of isomonodromic $2 \times 2$ Fuchsian systems is discussed thoroughly in [27].

\section{The COMputational Method}

Here we briefly recall the straightforward method for computation of almost Belyi coverings from $\mathbb{P}^{1}$ to $\mathbb{P}^{1}$ and present an improved method that uses differentiation. To distinguish the two projective curves, we write the coverings as $\mathbb{P}_{x}^{1} \rightarrow \mathbb{P}_{z}^{1}$, where $x$ and $z$ denote the rational parameters of the projective lines above and below, respectively. We assume that the three ramification loci indicated in the $R_{4}$-notation are $z=0, z=1, z=\infty$, in this order. We refer to the simple ramification point in the fourth ramified fiber as the extra ramification point.

Let $n$ denote the degree of the covering. By the Hurwitz genus formula [14, Corollary IV.2.4], the number of distinct points above $\{0,1, \infty\} \subset \mathbb{P}_{z}^{1}$ must be $n+3$ for an almost Belyi covering (and $n+2$ for a Belyi map); see [18, Proposition 2.1] or [29, Lemma 2.5].

The straightforward method to compute an almost Belyi covering $z(x)=\varphi(x)$ with a given ramification pattern is to write an ansatz of the form

$$
\varphi(x)=\frac{F}{H}, \quad \varphi(x)-1=\frac{P}{H},
$$

where $F, H, P$ are general polynomials in $x$ of the factorized form determined by the respective partition of $n$. Specifically, the polynomials have the form $C_{0} \prod_{j=1}^{n} Q_{j}^{j}$, where $C_{0}$ is a constant (yet undetermined), and each $Q_{j}$ is a monic general polynomial of degree equal to the number of parts $j$ in the respective partition. Of course, polynomials of degree zero can be skipped. In the resulting coverings, all polynomials $Q_{j}$ must have mutually distinct and simple roots. To avoid redundancy, we assume that $H$ is a monic polynomial, and pick 3 of the $x$-point 2 as $x=\infty$, $x=0$ and $x=1$. Expression (2.1) leads to the polynomial identity $F=P+H$; by expanding the polynomials and comparing the terms to the powers of $x$ one gets a set of polynomial equations.

This straightforward method was extensively used in [18, [19] to compute Belyi maps and almost Belyi coverings of degree up to 12. Those coverings were applied

\footnotetext{
${ }^{2}$ Strictly speaking, the $x$-points for almost Belyi coverings are curves, or one-dimensional branches of a generic family, parametrized by an isomonodromy parameter $t$ or other parameter, since the Hurwitz spaces for almost Belyi maps are one-dimensional. For simplicity, we ignore the dimensions introduced by such parameters and consider a one-dimensional Hurwitz space as a generic point.
} 
to compute algebraic transformations of Gauss hypergeometric functions, or to compute algebraic Painlevé VI functions. However, the amount of computations with the straightforward method grows quickly for larger $n$. In particular, the number of variables and the algebraic degree of the initial equations grow linearly with $n$. The polynomial system may have many degenerate (or parasitic [20]) solutions, when the rational expression in (2.1) can be simplified to a rational function of lower degree. The computation of higher degree coverings with the straightforward method is hardly possible even with modern computers. Our three coverings were too hard to compute in reasonable time with available PCs.

Equations of smaller degree for the undetermined coefficients can be obtained by considering derivatives of $\varphi(x)$. According to Couveignes [8, Fricke was probably the first to use differentiation to investigate highly ramified maps. More recently, differentiation was used for the investigation of dessins d'enfant in [24, [25], 32] and other works. Specifically for computational purposes, differentiation was used in [15] and [30] in similar ways.

A systematic procedure for the computation of Belyi maps with differentiation is formulated in [30, Section 3]. The main trick is to consider logarithmic derivatives of $\varphi$ and $\varphi-1$ from (2.1). For instance, the denominator of $\varphi^{\prime} / \varphi$ is the product of all factors of $F$ and $H$, to the power 1 . The numerator is the product of all factors of $P$, with the powers diminished by 1 . This gives equations of smaller degree, and easy possibilities for elimination. Typically, the degree of the equations is diminished by the number of distinct points in a corresponding ramified fiber. Compared with the computation of Belyi maps, the only adaptation for almost Belyi coverings is that numerators of the logarithmic derivatives have an additional degree 1 factor coming from the extra ramified point.

In this paper, we apply the method in [30, Section 3] for the computation of almost Belyi coverings with the ramification types (1.1)-(1.3). In the following section, we present the computational steps specifically for ramification pattern (1.1) quite in detail. Having demonstrated that example, we present computations for the other two ramification patterns in lesser detail in the routine steps, while concentrating rather on additional heuristic means of solving the obtained systems of equations. In particular, Section 5 uses modular methods for finding coverings with ramification pattern (1.3). Our examples show that computational complexity depends not only on the degree of the covering, but also on the geometric complexity of the solutions (apparently, the geometry is more complicated when the degree is prime), or the number of irreducible components of the solutions. Accordingly, different heuristic tricks can be useful for different coverings.

Here we make a few comments on the fields of definition and dimension of Hurwitz spaces. Whether we use the straightforward method or logarithmic derivatives, the algebraic equations for the coverings (or Hurwitz spaces) are defined over $\mathbb{Q}$. For Belyi functions, the solutions (up to fractional-linear transformations) are isolated points, generally defined over an algebraic extension of $\mathbb{Q}$. The field extension may depend on the fractional-linear normalization of fixing the points $x=\infty, x=0$, $x=1$. To be certain of a minimal $\mathbb{Q}$-extension, one may choose to fix the points of $\mathbb{P}_{x}^{1}$ where the ramification order is different from other ramification orders in the same fiber. The central question in the theory of dessins d'enfant is how the 
Galois group of $\overline{\mathbb{Q}} / \mathbb{Q}$ acts on Belyi coverings (with necessarily the same ramification pattern) or dessins d'enfant.

General almost Belyi coverings are parameterized by algebraic curves [33, [18]. If so preferred, one may consider them as one-dimensional families of almost Belyi coverings. The genus of parameterizing curves may depend on the normalization. To get a modelling curve of minimal genus, one strives to fix the points with "isolated" ramification orders as $x=\infty, x=0, x=1$, like in the zero-dimensional case. But the equation system can be simpler if we adopt the strategy of choosing the points with the highest ramification orders as $x=\infty, x=0, x=1$. This situation is demonstrated in Section 4.

\section{The DEGREe 12 COVERING}

Here we compute the generic pull-back covering with the ramification type

$$
R_{4}(3+3+3+1+1+1|2+2+2+2+2+2| 5+5+2) .
$$

Relatively speaking, this is a warm-up example.

By our conventions, the three partitions of 12 specify the ramification orders above $z=0, z=1$ and $z=\infty$, respectively. We choose the simple ramified point above $z=\infty$ as $x=\infty$, and the simple ramified point above the fourth $z$-point as $x=0$. We do not fix $x=1$, so there will be the torus action $x \mapsto \lambda x$ on the defining equations. The equations are expected to be weighted-homogeneous, and the Hurwitz space (if irreducible) should be a curve in a weighted-projective space of minimal possible genus.

We write the ansatz

$$
\varphi_{12}(x)=C_{0} \frac{F^{3} G}{H^{5}}, \quad \varphi_{12}(x)-1=C_{0} \frac{P^{2}}{H^{5}},
$$

where

$$
\begin{aligned}
F & =x^{3}+a_{1} x^{2}+a_{2} x+a_{3}, \\
G & =x^{3}+b_{1} x^{2}+b_{2} x+b_{3}, \\
H & =x^{2}+c_{1} x+c_{2}, \\
P & =x^{6}+p_{1} x^{5}+p_{2} x^{4}+p_{3} x^{3}+p_{4} x^{2}+p_{5} x+p_{6}
\end{aligned}
$$

are polynomials whose roots are the other $x$-points above $z \in\{0,1, \infty\}$. In particular, the roots of $F, H, P$ are the remaining ramified points. The roots of $F, G, H$, $P$ must be mutually distinct and simple. Besides, $C_{0}=\lim _{x \rightarrow \infty} \varphi_{12}(x) / x^{2}$ is a yet undetermined constant. The straightforward method would utilize the following consequence of (3.2):

$$
F^{3} G=P^{2}+\frac{1}{C_{0}} H^{5}
$$

Following [30, Section 3], we obtain simpler equations in the coefficients of $F$, $G, H, P$ by considering the logarithmic derivatives of $\varphi_{12}(x)$ and $\varphi_{12}(x)-1$. It is not hard to figure out the zeroes and poles of the logarithmic derivatives:

$$
\frac{\varphi_{12}^{\prime}}{\varphi_{12}}=C_{1} \frac{x P}{F G H}, \quad \frac{\left(\varphi_{12}-1\right)^{\prime}}{\varphi_{12}-1}=C_{2} \frac{x F^{2}}{H P} .
$$

Here $C_{1}=C_{2}=2$ by local considerations at $x=\infty$. One may generally notice that if $x=\infty$ is chosen above $z=\infty$ in a setting such as (3.2), the constants in the 
logarithmic derivative expressions like in (3.4) are equal to the ramification order at $x=\infty$.

Comparison of the numerators in (3.4) gives the following identities:

$$
2 x P=3 F^{\prime} G H+F G^{\prime} H-5 F G H^{\prime}, \quad 2 x F^{2}=2 P^{\prime} H-5 P H^{\prime} .
$$

The same type of expressions is obtained in computations in [15]. After expanding the polynomial expressions and collecting the terms to the powers of $x$ in (3.5), the first identity gives the following equations:

$$
\begin{aligned}
2 p_{1} & =7 c_{1}+b_{1}-a_{1}, \\
2 p_{2} & =12 c_{2}+6 b_{1} c_{1}+4 a_{1} c_{1}-2 a_{1} b_{1}-4 a_{2}, \\
\ldots & \cdots \\
2 p_{6} & =6 a_{1} b_{3} c_{2}+4 a_{2} b_{2} c_{2}-2 a_{2} b_{3} c_{1}+2 a_{3} b_{1} c_{2}-4 a_{3} b_{2} c_{1}-10 a_{3} b_{3}, \\
0 & =3 a_{2} b_{3} c_{2}+a_{3} b_{2} c_{2}-5 a_{3} b_{3} c_{1} .
\end{aligned}
$$

The second identity gives the equations

$$
\begin{aligned}
4 a_{1} & =7 c_{1}, \\
4 a_{2}+2 a_{1}^{2} & =12 c_{2}+5 c_{1} p_{1}-2 p_{2}, \\
\cdots & \cdots \cdots \\
2 a_{3}^{2} & =4 c_{2} p_{4}-3 c_{1} p_{5}-10 p_{6}, \\
0 & =2 c_{2} p_{5}-5 c_{1} p_{6} .
\end{aligned}
$$

The new equations are sufficient, since they are derived from necessary conditions. They have smaller algebraic degree and have less degenerate solutions. They can be solved even by brute force with Maple's routine solve. More systematically, one may use elimination or Gröbner basis techniques. The system is overdetermined, but superfluous equations only help the Gröbner basis computations. As mentioned, the equations are weighted homogeneous; specifically,

$$
\operatorname{deg} a_{j}=\operatorname{deg} b_{j}=\operatorname{deg} c_{j}=\operatorname{deg} p_{j}=j .
$$

The variables $p_{i}$ can be directly eliminated using the first set of equations. This can be done similarly for any covering problem with a fiber of only simple ramified points (with the ramification order 2) plus possibly one non-ramified point. Notice also that the second set of equations does not contain the $b_{i}$ 's. There is a dependence between the first two equations in (3.6) and the first two equations in (3.7).

A straightforward way to get the result is the following. Using the first equation in (3.7), we eliminate $a_{1}$. Then all equations are (still) linear in the $b_{i}$ 's and $p_{i}$ 's. We actually get 12 linearly independent equations in these 9 variables. These variables can be eliminated using determinants 3 or syzygie:4. We get

\footnotetext{
${ }^{3} \mathrm{~A}$ brute way to eliminate the 9 variables is to pick 9 (out of the 12 ) equations, solve them in the 9 variables, and substitute into the remaining equations. This is equivalent to the computation of $10 \times 10$ determinants, with polynomial entries in $c_{1}, c_{2}, a_{2}, a_{3}$. In the particular case, this method typically gives equations of degree 4 or 5 in $a_{3}$ alone.

${ }^{4}$ Here we mean syzygies between the 12 vectors in the rank 9 free module over $\mathbb{Q}\left[c_{1}, c_{2}, a_{2}, a_{3}\right]$, with the vector components equal to the corresponding coefficients of the 9 variables. When the syzygies are applied to the 12 equations, the 9 variables will be eliminated. In the particular case, we can get one equation of weighted degree 11 in $c_{1}, c_{2}, a_{2}, a_{3}$ in this way, and 3 independent equations of degree 12 .
} 
weighted homogeneous equations in $c_{1}, c_{2}, a_{2}, a_{3}$. We just have to eliminate one more variable 5 to get an equation for the Hurwitz curve. A single such equation in 3 weighted-homogeneous variables is likely to have large degree and superfluous factors. To avoid investigating all factors, we suggest computing two or more such equations, and consider only their common factors. It turns out that only the following factor gives a non-degenerate solution.

$$
160 a_{2}^{2} c_{1}^{2}+6912 a_{2} c_{2}^{2}-2256 a_{2} c_{1}^{2} c_{2}-188 a_{2} c_{1}^{4}+103680 c_{2}^{3}-81936 c_{1}^{2} c_{2}^{2}+20328 c_{1}^{4} c_{2}-1421 c_{1}^{6} .
$$

This weighted-homogeneous polynomial defines a curve of genus 0 . We can normalize $c_{1}=1$, and parameterize as follows:

$$
c_{2}=\frac{(2 t+1)(5 t+16)}{48 t}, \quad a_{2}=-\frac{(2 t+5)\left(15 t^{2}+25 t+16\right)}{16 t} .
$$

Going back, we consequently find parametric expressions for $a_{3}$ (and immediately for $a_{1}$ ), and then for the 9 variables $b_{i}$ and $p_{i}$. To find the constant $C_{0}$, one can use (3.3) evaluated at any $x \in \mathbb{Q}$.

To write the generic solution more compactly, we renormalize $x \mapsto x / 4$ and multiply the polynomials $F, G, H$ by some expressions in $t$. Here is the covering:

$$
\varphi_{12}(x)=C_{12} \frac{F_{12}^{3} G_{12}}{H_{12}^{5}},
$$

where

$$
\begin{aligned}
C_{12}= & -\frac{t^{2}\left(10 t^{2}+25 t+16\right)}{16(3 t+4)^{7}} \\
F_{12}= & 2 t x^{3}+14 t x^{2}-2(2 t+5)\left(15 t^{2}+25 t+16\right) x-(2 t+5)(5 t+16)\left(t^{2}+10 t+6\right), \\
G_{12}= & 50 t\left(10 t^{2}+25 t+16\right) x^{3}-30 t\left(14 t^{3}-18 t^{2}-105 t-80\right) x^{2} \\
& -6(5 t+16)(2 t+1)\left(20 t^{3}+35 t^{2}+3 t-16\right) x \\
& -(2 t+1)^{2}(5 t+16)^{2}\left(5 t^{2}+10 t+6\right), \\
H_{12}= & 3 t x^{2}+12 t x+(2 t+1)(5 t+16) .
\end{aligned}
$$

We have

$$
1-\varphi_{12}(x)=\frac{1}{16(3 t+4)^{7}} \frac{P_{12}^{2}}{H_{12}^{5}}
$$

\footnotetext{
${ }^{5}$ Here resultants can be used. In our computations, even if we took two equations of degree $4-5$ in $a_{3}$, Maple 9.5 computed a resultant with respect to $a_{3}$ in 15-30 seconds.

${ }^{6}$ Degenerate solutions are those for which the polynomials $F, G, H$ have multiple or common roots, or have the root $x=0$. Consequently, the factors such as $a_{3}, b_{3}, c_{2}$ or $c_{1}^{2}-4 c_{2}$ can be ignored. It might even be useful to search actively for polynomials divisible by the degeneracy factors, so that after dividing them out we possibly get polynomials of low degree. In Section 5 , we systematically search for polynomials divisible by two resultants that define degeneracy for the ramification pattern (1.3).
} 
where

$$
\begin{aligned}
P_{12}= & 20 t^{3}\left(10 t^{2}+25 t+16\right) x^{6}-12 t^{3}\left(7 t^{3}-184 t^{2}-490 t-320\right) x^{5} \\
& +60 t^{2}\left(4068 t^{3}+4048 t+1024+200 t^{5}+5885 t^{2}+1386 t^{4}\right) x^{4} \\
& +20 t^{2}\left(37627 t^{4}+100300 t^{3}+137092 t^{2}+6415 t^{5}+250 t^{6}+92992 t+24576\right) x^{3} \\
& -60 t(5 t+16)\left(200 t^{7}+1260 t^{6}+3052 t^{5}+3248 t^{4}+1126 t^{3}-137 t^{2}+256 t+256\right) x^{2} \\
& -30 t(5 t+16)^{2}\left(100 t^{7}+740 t^{6}+2289 t^{5}+3780 t^{4}+3600 t^{3}+2040 t^{2}+700 t+128\right) x \\
& -(2 t+1)(5 t+16)^{3}\left(100 t^{7}+740 t^{6}+2289 t^{5}+3780 t^{4}+3600 t^{3}+2040 t^{2}+700 t+128\right) .
\end{aligned}
$$

\section{The DEGReE 11 COVERING}

Here we compute the generic pull-back covering with the ramification type

$$
R_{4}(3+3+3+1+1|2+2+2+2+2+1| 5+5+1) .
$$

To get a Hurwitz curve of minimal genus, we choose the non-ramified point above $z=\infty$ as $x=\infty$, the non-ramified point above $z=1$ as $x=0$, and the simple ramified point above the fourth $z$-point as $x=1$. Accordingly, we write the ansatz

$$
\varphi_{11}(x)=C_{0} \frac{F^{3} G}{H^{5}}, \quad \varphi_{11}(x)-1=C_{0} \frac{x P^{2}}{H^{5}},
$$

where $F, G, H, P$ are polynomials of degrees $3,2,2$ and 5 , respectively. Zeroes and poles of the logarithmic derivatives of $\varphi_{11}(x)$ and $\varphi_{11}(x)-1$ are easy to figure out, as in (3.4). The method gives the following identities:

$$
\begin{aligned}
2(x-1) P & =3 F^{\prime} G H+F G^{\prime} H-5 F G H^{\prime}, \\
2(x-1) F^{2} & =2 x P^{\prime} H-5 x P H^{\prime}+P H .
\end{aligned}
$$

However, the resulting equations in the coefficients of $F, G, H, P$ are still too complicated to solve by direct elimination or Gröbner basis techniques.

Equations of smaller algebraic degree are obtained if we adopt the strategy to normalize the points with highest ramification orders as $x=\infty, x=0$, etc. (But then the Hurwitz space can have non-minimal genus.) In particular, we choose the two points of ramification order 5 (above $z=\infty$ ) as $x=0$ and $x=\infty$. We choose the extra ramified point above the fourth locus as $x=1$, as just above. Then the non-ramified points above $z=\infty$ and $z=1$ are undetermined. We denote their location as $x=c_{1}$ and $x=c_{2}$, respectively. That gives the following ansatz:

$$
\widetilde{\varphi}_{11}(x)=\widetilde{C}_{0} \frac{F^{3} G}{x^{5}\left(x-c_{1}\right)}, \quad \widetilde{\varphi}_{11}(x)-1=\widetilde{C}_{0} \frac{P^{2}\left(x-c_{2}\right)}{x^{5}\left(x-c_{1}\right)},
$$

where

$$
\begin{aligned}
& F=x^{3}+a_{1} x^{2}+a_{2} x+a_{3}, \\
& G=x^{2}+b_{1} x+b_{2}, \\
& P=x^{5}+p_{1} x^{4}+p_{2} x^{3}+p_{3} x^{2}+p_{4} x+p_{5},
\end{aligned}
$$

and $C_{0}=\lim _{x \rightarrow \infty} \widetilde{\varphi}_{11}(x) / x^{5}$ is an undetermined constant. The fractional-linear transformation on $\mathbb{P}_{x}^{1}$ from (4.5) to (4.2) is

$$
x \mapsto \frac{c_{1}\left(c_{2}-1\right) x+c_{2}\left(1-c_{1}\right)}{\left(c_{2}-1\right) x+1-c_{1}} .
$$


Consideration of logarithmic derivatives of $\widetilde{\varphi}_{11}(x)$ and $\widetilde{\varphi}_{11}(x)-1$ gives the following equations:

$$
\begin{aligned}
5(x-1) P & =3 x\left(x-c_{1}\right) F^{\prime} G+x\left(x-c_{1}\right) F G^{\prime}-\left(6 x-5 c_{1}\right) F G, \\
5(x-1) F^{2} & =2 x\left(x-c_{1}\right)\left(x-c_{2}\right) P^{\prime}-\left(5 x^{2}-4 c_{1} x-6 c_{2} x+5 c_{1} c_{2}\right) P .
\end{aligned}
$$

As in the previous example, collecting terms in like powers of $x$ gives a system of equations in the $a_{i}$ 's, $b_{i}$ 's, $c_{i}$ 's and $p_{i}$ 's. First we pick up the following 8 equations: the terms to the powers $5,4,2,1,0$ of $x$ in (4.7), and the terms to the powers 6,1 , 0 in (4.8). Using these equations, we eliminate the $a_{i}$ 's and $p_{i}$ 's. Formally, there are 2 solution components, but the one with $a_{3}=0$ has to be discarded as degenerate 7 We obtain several non-homogeneous equations in the 4 variables $b_{1}, b_{2}, c_{1}, c_{2}$. To get equations for the Hurwitz curve in two variables, we eliminate $b_{1}, b_{2}$ by picking up equations of minimal degree in them and using resultants. As in the previous section, we can compute several resultant polynomials (in $c_{1}, c_{2}$ only) and consider their common divisors as candidate models for the Hurwitz curve. It turns out that there is possibly only one component of non-degenerate solutions. It is described by a polynomial factor of degree 15 in $c_{1}, c_{2}$.

Let $Q$ denote the degree 15 factor. It apparently defines the Hurwitz space for the desired almost Belyi map with chosen normalization. The degree of $Q$ in $c_{1}$ alone is 12 ; the degree in $c_{2}$ is just 6 . According to Maple's package algcurves, $Q$ defines a curve of genus 3. Luckily, the curve is hyperelliptic. Improvised computations produced the following Weierstrass model:

$$
w^{2}=\left(3 t^{2}+3 t+2\right)\left(27 t^{6}+71 t^{5}+130 t^{4}+140 t^{3}+120 t^{2}+64 t+32\right) .
$$

The variables $c_{1}$ and $c_{2}$ can be parameterized as

$$
\begin{aligned}
& c_{1}=\frac{27 t^{6}+67 t^{5}+116 t^{4}+118 t^{3}+94 t^{2}+46 t+20+w\left(3 t^{2}+2 t+2\right)}{2(t+2)\left(t^{2}+1\right)\left(2 t^{2}+3 t+3\right)} \\
& c_{2}=\frac{1107 t^{12}+7641 t^{11}+26055 t^{10}+L_{1}-w\left(9 t^{3}+19 t^{2}+13 t+7\right)\left(3 t^{5}+15 t^{4}+15 t^{3}+45 t^{2}+40 t+26\right)}{2(t+2)(3 t+1)^{3}\left(t^{2}+1\right)\left(2 t^{2}+3 t+3\right)^{2}\left(5 t^{2}+4 t+3\right)}
\end{aligned}
$$

where

$L_{1}=59035 t^{9}+99475 t^{8}+130463 t^{7}+138619 t^{6}+121015 t^{5}+87870 t^{4}+51600 t^{3}+23798 t^{2}+7574 t+1460$.

Using these expressions and the equations in $b_{1}, b_{2}, c_{1}, c_{2}$, we parameterize $b_{1}$ and $b_{2}$ :

$$
\begin{aligned}
& b_{1}=\frac{\left(27 t^{5}-45 t^{4}-190 t^{3}-360 t^{2}-360 t-216\right)\left(L_{2}+w(t+7)\left(6 t^{2}+3 t+2\right)\right)}{32(3 t+1)^{3}\left(t^{2}+1\right)\left(2 t^{2}+3 t+3\right)^{2}\left(5 t^{2}+4 t+3\right)} \\
& b_{2}=-\frac{2916 t^{14}+11124 t^{13}+191673 t^{12}+L_{3}+w(t+7)\left(6 t^{2}+3 t+2\right) L_{2}}{32(3 t+1)^{3}\left(t^{2}+1\right)^{2}\left(2 t^{2}+3 t+3\right)\left(5 t^{2}+4 t+3\right)^{2}}
\end{aligned}
$$

\footnotetext{
${ }^{7}$ Here is a stepwise course of elimination. The zeroth powers of $x$ in (4.7)-(4.8), or substitution $x=0$, give the equations $5 p_{5}+5 c_{1} b_{2} a_{3}=0$ and $5 a_{3}^{2}=5 c_{1} c_{2} p_{5}$. It is easy to eliminate $a_{3}, p_{5}$. We must ignore solutions with $a_{3}=0$, so we are left with $a_{3}=-c_{1}^{2} c_{2} b_{2}$. Next we consider the coefficients of the first powers in $x$ in 4.7 -4.8), and eliminate $a_{2}, p_{4}$. We get, in particular,

$$
a_{2}=\frac{c_{1}}{11}\left(5 c_{1} b_{2}+12 c_{2} b_{2}-3 c_{1} c_{2} b_{1}-10 c_{1} c_{2} b_{2}\right) .
$$

Similarly, we consider the coefficients of the highest degrees (5 and 6, respectively) of $x$ in 4.7)(4.8), and eliminate $a_{1}, p_{1}$. We get, in particular, $a_{1}=\left(3 b_{1}-12 c_{1}-5 c_{2}+10\right) / 11$. Having expressed the $a_{i}$ 's and $p_{1}, p_{4}, p_{5}$ just in terms of $b_{i}$ 's and $c_{i}$ 's, the remaining equations are linear in $p_{2}, p_{3}$. These two $p_{i}$ 's can be eliminated using discriminants or syzygies, as in the previous section.
} 
where $L_{2}=54 t^{7}-297 t^{6}-682 t^{5}-1145 t^{4}-970 t^{3}-712 t^{2}-304 t-104$, and

$$
\begin{aligned}
L_{3}= & 764136 t^{11}+1953326 t^{10}+3445832 t^{9}+4698345 t^{8}+5040404 t^{7}+4425220 t^{6} \\
& +3147872 t^{5}+1833840 t^{4}+840864 t^{3}+301376 t^{2}+74176 t+11680 .
\end{aligned}
$$

Here we stop computations on the hyperelliptic curve. Using the fractional-linear transformation (4.6), we express the polynomials $H$ and $G$ in (4.2) as follows:

$$
\begin{aligned}
& H=x^{2}-\frac{\left(c_{1}-1\right)\left(c_{1}+c_{2}\right)}{c_{1}\left(c_{2}-1\right)} x+\frac{c_{2}\left(c_{1}-1\right)^{2}}{c_{1}\left(c_{2}-1\right)^{2}} \\
& G=x^{2}-\frac{\left(c_{1}-1\right)\left(2 c_{1} c_{2}+c_{1} b_{1}+c_{2} b_{1}+2 b_{2}\right)}{\left(c_{2}-1\right)\left(c_{1}^{2}+c_{1} b_{1}+b_{2}\right)} x+\frac{\left(c_{1}-1\right)^{2}\left(c_{2}^{2}+c_{2} b_{1}+b_{2}\right)}{\left(c_{2}-1\right)^{2}\left(c_{1}^{2}+b_{1} c_{1}+b_{2}\right)}
\end{aligned}
$$

When we write these coefficients in terms of $t, w$, the square root $w$ conveniently disappears. Hence all coefficients are just rational functions in $t$. We checked that the algebraic relations between the coefficients of $H$ define an irreducible curve of degree 13 (and genus 0, as parameterized by $t$ ). The projective degree of the parameterization by $t$ is 13 as well, so the parameterization is minimal.

We go back to the equations induced by (4.3)-(4.4), but now knowing parametric expressions for 4 variables. It is then straightforward to find parametric expressions for the remaining coefficients of $F$ in (4.2). The final expression for $\varphi_{11}$ can be simplified by the renormalization

$$
x \mapsto \frac{3 t^{5}+15 t^{4}+15 t^{3}+45 t^{2}+40 t+26}{\left(3 t^{2}+2 t+2\right)\left(5 t^{2}+4 t+3\right)} x .
$$

In this normalization, the extra ramified point is not fixed as $x=1$. The final expression can be written as:

$$
\varphi_{11}(x)=C_{11} \frac{F_{11}^{3} G_{11}}{H_{11}^{5}},
$$

where

$$
\begin{aligned}
C_{11}= & -\frac{\left(2 t^{2}+3 t+3\right)(3 t+1)^{2}}{108}, \\
F_{11}= & x^{3}-\frac{4941 t^{6}+13122 t^{5}+19905 t^{4}+17820 t^{3}+10795 t^{2}+3962 t+879}{(3 t+1)^{2}\left(2 t^{2}+3 t+3\right)} x^{2} \\
& +\frac{(3 t+1)\left(432 t^{5}+570 t^{4}+330 t^{3}-265 t^{2}-340 t-151\right)}{2 t^{2}+3 t+3} x+3\left(2 t^{2}+3 t+3\right)(3 t+1)^{4}, \\
G_{11}= & \left(3 t^{2}+2 t+2\right)^{2} x^{2} \\
& +\frac{27 t^{10}+270 t^{9}+945 t^{8}+2160 t^{7}+2745 t^{6}+1926 t^{5}-5 t^{4}-1340 t^{3}-1440 t^{2}-720 t-216}{\left(2 t^{2}+3 t+3\right)(3 t+1)^{2}} x \\
& -4(3 t+1)\left(2 t^{2}+3 t+3\right), \\
H_{11}= & \left(5 t^{2}+4 t+3\right) x^{2}+\frac{135 t^{6}+396 t^{5}+715 t^{4}+790 t^{3}+610 t^{2}+280 t+82}{2 t^{2}+3 t+3} x \\
& +\left(2 t^{2}+3 t+3\right)(3 t+1)^{3} .
\end{aligned}
$$

Let $P_{11}$ denote the degree 5 polynomial such that

$$
1-\varphi_{11}(x)=\frac{1}{108\left(2 t^{2}+3 t+3\right)} \frac{x P_{11}^{2}}{H_{11}^{5}} .
$$

We have $P_{11}=\left(3 t^{2}+2 t+2\right)\left(2 t^{2}+3 t+3\right)(3 t+1) x^{5}+\ldots$.

As we see, the degree 11 covering is more complicated than the degree 12 covering of Section 3 . Apparently the geometry of prime degree coverings is more complex. 


\section{The Degree 20 COVERINGS}

Here we compute generic pull-back coverings with the ramification type

$$
R_{4}(5+5+5+5|2+2+2+2+2+2+2+2+2+2| 3+3+3+3+3+2+1+1+1) .
$$

By fractional-linear transformations, we fix the simple ramified point above $z=\infty$ as $x=\infty$, and we choose the extra ramified point as $x=0$.

The ansatz is

$$
\varphi_{20}(x)=C_{0} \frac{F^{5}}{G^{3} H}, \quad \varphi_{20}(x)-1=C_{0} \frac{P^{2}}{G^{3} H},
$$

where

$$
\begin{aligned}
F & =x^{4}+a_{1} x^{3}+a_{2} x^{2}+a_{3} x+a_{4}, \\
G & =x^{5}+b_{1} x^{4}+b_{2} x^{3}+b_{3} x^{2}+b_{4} x+b_{5}, \\
H & =x^{3}+c_{1} x^{2}+c_{2} x+c_{3}, \\
P & =x^{10}+p_{1} x^{9}+p_{2} x^{8}+\cdots+p_{9} x+p_{10},
\end{aligned}
$$

and $C_{0}=\lim _{x \rightarrow \infty} \varphi(x) / x^{2}$ is an undetermined constant. Consideration of the logarithmic derivatives of $\varphi_{20}(x)$ and $\varphi_{20}(x)-1$ gives the following identities:

$$
2 x P=5 F^{\prime} G H-3 F G^{\prime} H-F G H^{\prime}, \quad 2 x F^{4}=2 P^{\prime} G H-3 P G^{\prime} H-P G H^{\prime} .
$$

After expanding and collecting terms in like powers of $x$, we get a system of equations in the coefficients of $F, G, H, P$. Since we do not fix $x=1$, the equations are weighted homogeneous, with the same grading as formulated in (3.8).

There are many possibilities for eliminating variables from the equation system. For example, one may use the first 10 equations of the first identity to eliminate all the $p_{i}$ 's. Then we can use the first equation from the second group to eliminate $b_{1}$; the subsequent equation turns out to be void. The next 3 equations allow us to eliminate $b_{3}, b_{4}$ and $b_{5}$. But still, there are too many variables left to solve the system by force.

Our strategy is the following. We solve the equations modulo several large primes, isolate non-degenerate solutions, and try to lift them to the characteristic 0 . The principle aim is to derive modular polynomial equations which characterize only non-degenerate solutions. When lifted to $\mathbb{Q}$, those equations are expected to have low degree and rather small coefficients, because they would contain information only about relevant solutions. Eventually, it turns out that there are a few connected components of non-degenerate solutions; we are able to separate them on the modular level, so that each lifted equation system describes only one connected component. We effectively avoid intermediate computations with huge $\mathbb{Q}$-coefficients, and consider over $\mathbb{Q}$ only those equation systems which describe isolated components of the generic solution. We are able to get just a few new polynomial equations over $\mathbb{Q}$ of low degree, but that is just enough for a breakthrough simplification of the original system.

We use the computer algebra package Singular 13, well suited for ring-theoretic manipulation modulo large primes. For a prime number $p$, let $\mathbb{F}_{p}$ denote the finite field with $p$ elements. We did computations modulo these primes:

$$
p \in\{32003,31991,31981,31973,31963\} .
$$


The solutions were found after considering the first 4 primes, while computations modulo 31963 were done for checking purposes only.

We use the weighted grading as formulated in (3.8). To be able to discard degenerate solutions, let $Q$ denote the resultant of $F$ and $G$ with respect to $x$, and let $Z$ denote the resultant of $F$ and $H$. Their weighted degrees are 20 and 12 , respectively. A solution of the original equation system is degenerate if and only if $Q=0$ or $Z=0$.

For each prime number $p$ from (5.5), we do computations in two rings:

$$
\begin{aligned}
& R_{1}=\mathbb{F}_{p}\left[a_{1}, a_{2}, a_{3}, a_{4}, b_{2}, c_{1}, c_{2}, c_{3}, Z\right], \\
& R_{2}=\mathbb{F}_{p}\left[a_{1}, a_{2}, a_{3}, a_{4}, b_{2}, c_{1}, c_{2}, c_{3}, Z, Q\right] .
\end{aligned}
$$

We assume that $b_{1}, b_{3}, b_{4}, b_{5}$ and the $p_{i}$ 's are eliminated from the original system. Let $J_{0}$ denote the graded ideal in $R_{2}$ generated by the original polynomial equations (after the elimination) and by definitions of $Z$ and $Q$. We wish to find polynomials in $J_{0}$ divisible by $Z$ or $Q$, so that we could get equations of lower degree for nondegenerate solutions by dividing such polynomials by the factor $Z$ or $Q$.

Let $J_{1}$ denote the restriction of $J_{0}$ onto $R_{1}$. For the beginning, we compute a Gröbner basis for $J_{1}$ in $R_{1}$ with respect to the total degree reverse lexicographic ordering with

$$
a_{4} \succ c_{3} \succ a_{3} \succ c_{2} \succ b_{2} \succ a_{2} \succ c_{1} \succ a_{1} \succ Z \text {. }
$$

With this ordering, a (weighted) homogeneous polynomial is divisible by $Z$ if and only if the leading term is divisible by $Z$. Let $G_{1}$ denote the Gröbner basis for $J_{1}$. We actually computed $G_{1}$ up to bounded degree 25 . The computations were done on a Dell laptop computer with Pentium M, $1700 \mathrm{MHz}$ processor on the Windows XP platform.

The first element of $G_{1}$ divisible by $Z$ occurs in degree 24. (Computations up to this degree took 430 seconds.) Non-degenerate solutions should satisfy the other degree 12 factor. We have two options: either use the new degree 12 equation immediately and recompute the Gröbner basis through degrees 12 to 24 , or continue computations in degree 25 in the hope of finding more elements of $G_{1}$ divisible by $Z$. The second option appears to be more acceptable since its next step takes less time (510 versus 650 seconds). Besides, the more greedy strategy of using lowest degree new polynomials immediately leads to more frequent and lengthier recomputations of Gröbner bases. In general, one may try different tactical choices when making computations modulo the first few different primes, and then use the best options when computing modulo other primes.

Our computations are summarized in Table 1. Recall that the Hilbert series of a graded ring $R$ is the series $\sum_{j=0}^{\infty} h_{j} t^{j}$, where $h_{j}$ is the dimension (over the ground field) of the $j$ th graded part of $R$. We refer to the numbers $h_{j}$ as Hilbert dimensions.

In the first column of Table 1, we list the weighted degrees from 11 to 25 . The numbers in small font are the Hilbert dimensions for $R_{1}$ (i.e., the number of monomials of the weighted degrees in $R_{1}$ ). In the second column, we give the number of elements of $G_{1}$ up to each degree, and (in the small font) the Hilbert dimensions for the ring $R_{1} / J_{1}$. As mentioned, there is one $Z$-multiple in degree 24 and two independent $Z$-multiples in degree 25 ; this is indicated in the next-to-last 
TABLE 1. Statistics of modular computations

\begin{tabular}{|c|c|c|c|c|c|c|c|}
\hline \multirow{2}{*}{$\begin{array}{c}\text { Degree } \\
\text { (Hilbert dim) }\end{array}$} & \multicolumn{7}{|c|}{ Gröbner basis (re)computations } \\
\hline & $1 \mathrm{st}$ & 2nd & 3rd & 4th & 5 th & 6 th & 7 th \\
\hline 11 & 9 & 9 & 9 & 9 & 110 & 113 & 24 \\
\hline 704 & 613 & 613 & 613 & 613 & 508 & 496 & 108 \\
\hline 12 & 12 & 13 & 15 & 98 & 158 & 143 & 120 \\
\hline 1020 & 848 & 847 & 845 & 762 & 587 & 571 & 99 \\
\hline 13 & 16 & 19 & 21 & 104 & 165 & 144 & 138 \\
\hline 1432 & 1128 & 1124 & 1120 & 954 & 660 & 643 & 105 \\
\hline 14 & 23 & 27 & 30 & 268 & 169 & 149 & 139 \\
\hline 1998 & 1479 & 1469 & 1457 & 959 & 738 & 718 & 113 \\
\hline 15 & 32 & 40 & 47 & 328 & 178 & 154 & 141 \\
\hline 2724 & 1877 & 1853 & 1829 & 1022 & 811 & 790 & 119 \\
\hline 16 & 45 & 59 & 70 & 365 & 185 & 159 & 147 \\
\hline 3689 & 2347 & 2298 & 2248 & 1088 & 889 & 865 & 127 \\
\hline 17 & 63 & 88 & 106 & 374 & 199 & 169 & 154 \\
\hline 4906 & 2851 & 2759 & 2671 & 1185 & 962 & 937 & 133 \\
\hline 18 & 85 & 123 & 157 & 418 & 209 & 180 & 159 \\
\hline 6486 & 3414 & 3255 & 3099 & 1251 & 1040 & 1012 & 141 \\
\hline 19 & 123 & 178 & 229 & 433 & 229 & 197 & 160 \\
\hline 8448 & 3980 & 3720 & 3470 & 1348 & 1113 & 1084 & 148 \\
\hline 20 & 166 & 237 & 327 & 480 & 250 & 221 & \\
\hline 10943 & 4575 & 4174 & 3776 & 1414 & 1191 & 1160 & \\
\hline 21 & 236 & 346 & 484 & 506 & 286 & 256 & \\
\hline 14004 & 5129 & 4543 & 3950 & 1511 & 1264 & 1233 & \\
\hline 22 & 305 & 456 & 668 & 591 & 353 & 317 & \\
\hline 17827 & 5672 & 4854 & 3983 & 1577 & 1342 & 1312 & \\
\hline 23 & 427 & 610 & 1021 & 688 & 363 & 329 & \\
\hline 22464 & 6126 & 5042 & 3828 & 1674 & 1415 & 1390 & \\
\hline 24 & 535 & 852 & 1456 & & & 346 & \\
\hline 28173 & 6539 & 5177 & 3645 & & & 1478 & \\
\hline $\begin{array}{c}25 \\
35024\end{array}$ & $\begin{array}{l}\mathbf{6 9 5} \\
6837\end{array}$ & & & & & & \\
\hline New & $1+2$ & 2 & 83 & $4+97$ & $9+3$ & $1+2$ & $7+5+1$ \\
\hline Time (s) & 941 & 918 & 1367 & 520 & 142 & 93 & 2 \\
\hline
\end{tabular}

row, and by the boldface numbers in the table. Therefore we have 3 new suitable equations of degree 12 or 13 . Let $J_{2}$ denote the ideal generated by $J_{1}$ and the 3 new equations.

Subsequently, we compute the Gröbner basis for $J_{2}$ up to degree 24. Column 3 of Table 1 gives the same size statistics for $J_{2}$. This second run gives 2 new equations of degree 12. We iterate the procedure of adjoining new equations and recomputing the Gröbner basis until we don't see Gröbner basis elements with the leading monomial divisible by $Z$. As indicated by Table 1, the subsequent two runs give bonanzas of 83 and 101 new equations of degrees 10 to 12 . The same quantity and degree of new equations occurs modulo each chosen prime, which is a good indication. The statistics of Table 1 indicate the complexity of the computations in each run. In particular, the Hilbert dimensions indicate the size of the Gröbner basis elements in each degree. 
After the 6th run, we don't get Gröbner basis elements divisible by $Z$. Then we redo this run in the ring $R_{2}$; we use the same ordering as in (5.6) with additionally $Z \succ Q$. With this ordering, a homogeneous polynomial is divisible by $Q$ if and only if the leading term is divisible by $Q$. If the degree of the polynomial is less than $\operatorname{deg} Q=20$, we still have the same criterion for divisibility by $Z$. We do the computation in $R_{2}$ up to degree 24 , and we get one Gröbner basis element of degree 23 and two elements of degree 24 divisible by $Q$. After dropping the factor $Q$, we get an equation of degree 3 and two equations of degree 4 for nondegenerate solutions! We feed these equations into the current Gröbner basis in $R_{2}$; the following run gives a few equations divisible by $Z$ of degree up to 19 . After adding the new equations (of degree up to $19-12=7$ ), we get a stable Gröbner basis: 17 equations of degree up to 7, plus expressions for $Z$ and $Q$. This looks like the desired system of polynomial equations for non-degenerate solutions.

The computations with Singular were semiautomatic. For the first two primes, we tried a couple of strategies when to recompute a Gröbner basis. For computations modulo other primes we used empirically the most effective degree bounds. Those computations were automatic in principle; in particular, the 6th run was done directly in $R_{2}$. As mentioned, the new equations in the corresponding recomputations have the same degree (and leading monomials) modulo each chosen prime. Computations for one prime took about an hour.

Now we wish to lift the simplest new equations to characteristic 0. Modular lifting of Gröbner bases is considered by several authors; see, for example, 3] and further references. Strictly speaking, we do not know good bounds for the size of the $\mathbb{Q}$-coefficients. To be sure bounds must be huge, due to the suspected complexity of intermediate computations purely in $\mathbb{Q}$. Our whole idea is to escape the intermediate computations by considering over $\mathbb{Q}$ exclusively equations for non-degenerate solutions only. Eventually, we obtained correct solutions after using just the few primes and a few new lifted equations. At the end of this section we indicate a way to check for certain that there are no other solutions.

A straightforward way to lift equations to characteristic 0 is to lift the coefficients to rational numbers with smallest numerators and denominators. Modular reconstruction of rational numbers is a well-known problem; the basic algorithm is proved in [31]. A good indication that a lift of a polynomial is correct is that the denominators of the coefficients have the same factors. If the straightforward method did not work, we tried the LLL reduction algorithm [21 for the lattice generated by: the vector whose entries are the integer coefficients modulo the composite modulus, plus one extra zero component; and the vectors with two non-zero entries - the entry 1 at the extra component, and the composite modulus at some (and each) other component. In successful cases, the shortest LLL basis vector is shorter by several orders of magnitude than the other vectors, which is a convincing indication. We used the extra vector component because Maple's LLL routine requires linearly independent input.

As mentioned, each 6th run of modular computations gives one equation of (weighted) degree 3 and two equations of degree 4 . The degree 3 polynomials can 
be lifted quite easily. Up to a scalar factor, the result is convincingly

$$
\begin{array}{r}
\frac{625}{3} c_{3}-\frac{875}{4} a_{3}+11 c_{2} c_{1}+22 b_{2} c_{1}-\frac{643}{4} a_{2} c_{1}-\frac{99}{10} c_{1}^{3}-\frac{179}{4} a_{1} c_{2} \\
+\frac{44}{3} a_{1} b_{2}+\frac{369}{2} a_{2} a_{1}+\frac{143}{10} a_{1} c_{1}^{2}+\frac{3811}{60} a_{1}^{2} c_{1}-\frac{895}{12} a_{1}^{3} .
\end{array}
$$

The two polynomials of degree 4 can be reliably lifted from the obtained modular data as well. With the lifted polynomials, we can immediately eliminate $c_{3}$ and $a_{4}$. But further brute force computations seem to be too cumbersome still.

The final Gröbner basis for non-degenerate solutions appeared to have new equations of degree 5 or higher, but the modular data was not sufficient to lift them. We proceeded then to eliminate variables modulo the primes, so as to get modular equations in the minimal number of variables, which is 3 (weighted-homogeneous) variables to define the Hurwitz curves. These equations indicated that there are a few irreducible components of non-degenerate solutions. We suspected that factorized equations in 3 variables can be lifted easily; even if they have high degree, their coefficients can be expected to be rather simple.

Specifically, we used modular Gröbner basis computations with respect to an elimination ordering, still in Singular. After elimination, the equation in (only) $a_{1}$, $c_{1}, a_{2}$ has degree 14 modulo each prime in (5.5). It factors as follows: two factors of degrees 8 and 6 modulo 31991 and 31963; or three factors of degrees 8, 3, 3 modulo the other primes. This suggests that there are three families of non-degenerate solutions; one family corresponds to the degree 8 factors; the other two families should be conjugate over a quadratic extension of $\mathbb{Q}$. We succeeded in lifting the degree 8 and degree $6=3+3$ factors to $\mathbb{Q}$ using the LLL algorithm.

It turns out that the degree 6 polynomial factors over $\mathbb{Q}(\sqrt{-15})$ into two factors of degree 3 , as expected. One may check that the factors define genus 0 curves. The curves can be parameterized, and the other variables can be uniquely parameterized as well using the original equations and the three lifted equations of degree 3 or 4 . But we noticed a shortcut: ramification type (5.1) can be realized by a composition of two coverings of the types:

$$
R_{3}(3+\widehat{1}+\widehat{1}|5| 2+2+\widehat{1}) \quad \text { and } \quad R_{4}(3+1|2+1+1| 2+2) .
$$

The hats in the first expression indicate the ramification locus of the subsequent degree 4 covering. The coverings of these two types are known due to their application to Gauss hypergeometric functions and algebraic Painlevé VI functions, as we recall in Section 6 below. The (normalized generic) coverings for these ramification types are:

$$
\begin{aligned}
& \varphi_{5}(x)=\frac{(5-3 \sqrt{-15})(128 x+7+33 \sqrt{-15})^{5}}{8000 x(1024 x-781-171 \sqrt{-15})^{3}}, \\
& \varphi_{4}(z)=\frac{(t-3)^{3}(3 t-1)^{3}(z+1)(z+t)}{(t-1)^{2}\left((t-1)^{2} z+t(t+1)\right)(4 z+3(t+1))^{3}} .
\end{aligned}
$$

Note the appearance of $\sqrt{-15}$ in the first covering. The second covering has the parameter $t$ as it is an almost Belyi function. The composition $\varphi_{4} \circ \varphi_{5}(x)$ can be written in the form (5.2). We checked that the coefficients $a_{1}, c_{1}, a_{2}$ in this form parameterize the degree 6 polynomial factor. It follows that the two coverings implied by the degree 6 factor are $\varphi_{4} \circ \varphi_{5}$ and the conjugated version. 
The degree 8 factor in $a_{1}, c_{1}, a_{2}$ defines a genus 0 curve as well. Its parameterization gives rise (by the original equations and the three lifted equations of degree 3 or 4) to the following impressive solution of the covering problem:

$$
\begin{aligned}
(5.11) & \\
a_{1}= & 4\left(3 t^{4}+9 t^{3}+43 t^{2}+40 t+12\right), \\
a_{2}= & 6\left(182 t^{6}+728 t^{5}+2373 t^{4}+3584 t^{3}+2632 t^{2}+960 t+144\right), \\
a_{3}= & 4(t+1)\left(8 t^{2}+7 t+2\right)\left(1029 t^{5}+3246 t^{4}+9608 t^{3}+10224 t^{2}+4752 t+864\right), \\
a_{4}= & (5 t+6)\left(8 t^{2}+7 t+2\right)^{2}\left(1029 t^{5}+3246 t^{4}+9608 t^{3}+10224 t^{2}+4752 t+864\right), \\
b_{1}= & -\frac{1}{5}\left(4 t^{4}+12 t^{3}-869 t^{2}-892 t-276\right), \\
b_{2}= & -\frac{1}{5}\left(448 t^{6}+1756 t^{5}-59175 t^{4}-123710 t^{3}-101900 t^{2}-39144 t-6048\right), \\
b_{3}= & -\frac{1}{5}\left(8 t^{2}+7 t+2\right) \\
& \times\left(2352 t^{6}+9399 t^{5}-256240 t^{4}-577360 t^{3}-506160 t^{2}-204336 t-32832\right), \\
b_{4}= & -\frac{2}{5}\left(8 t^{2}+7 t+2\right)^{2}\left(56 t^{2}+79 t+34\right)\left(49 t^{4}+132 t^{3}-5184 t^{2}-4752 t-1296\right), \\
b_{5}= & -\frac{2}{5}\left(8 t^{2}+7 t+2\right)^{3}\left(49 t^{2}+76 t+36\right)\left(49 t^{4}+132 t^{3}-5184 t^{2}-4752 t-1296\right), \\
c_{1}= & \frac{1}{3}\left(100 t^{4}+300 t^{3}+507 t^{2}+388 t+108\right), \\
c_{2}= & \frac{1}{3}(5 t+6)\left(8 t^{2}+7 t+2\right)\left(140 t^{3}+307 t^{2}+308 t+108\right), \\
c_{3}= & \frac{1}{3}(5 t+6)^{2}\left(8 t^{2}+7 t+2\right)^{2}\left(49 t^{2}+76 t+36\right) .
\end{aligned}
$$

Let us denote by $\varphi_{20}(x)$ the covering defined by (5.2), (5.3) and (5.11). We refer to the other two solutions as compositions of the degree 4 and 5 coverings.

To summarize, we looked for the degree 20 coverings by modular methods, deliberately ignoring the size of the intermediate would be computations over $\mathbb{Q}$. It is theoretically possible that some generic solutions are missing, since they coincide with the derived solutions modulo each of the considered primes, or the original equation system is insufficient modulo those primes. This possibility has extremely low probability. Very likely, the set of "bad" primes consists of just a few small prime numbers. Regarding the application to Painlevé VI functions of the next section, we already know that there are exactly two algebraic solutions of $P_{\mathrm{VI}}(0,0,0,-2 / 3 ; t)$ from the work of Dubrovin and Mazzocco [11]: the Cube solution and the Great Dodecahedron solution. After an Okamoto transformation we have exactly two solutions of $P_{\mathrm{VI}}(1 / 3,1 / 3,1 / 3,1 / 3 ; t)$; they are obtainable from the composition $\varphi_{4} \circ \varphi_{5}(x)$ and the irreducible covering $\varphi_{20}(x)$, as we will see.

The canonical method for checking that there are certainly no other coverings with the ramification pattern (5.1) is combinatorial. Each branch of the Hurwitz space corresponds to a 4 -tuple of permuations of 20 elements, of cycle types $5+5+5+5,3+3+3+3+3+2+1+1+1$, etc. The braid group on 4 braids acts on the branches of the same connected component. There must be only three orbits of the braid group, giving three connected components of the Hurwitz space, corresponding to the composition $\varphi_{4} \circ \varphi_{5}(x)$, its complex conjugate, and $\varphi_{20}(x)$. This method is strict [8, 22, but it requires the computation of all permutations and combinations with the given cycle type and the identity product. More geometrically, one may introduce deformations of dessins d'enfant [18, 19], cacti [23] or similar geometric objects [6, pg. 105] that represent almost Belyi coverings in the same way as the usual dessins d'enfant correspond to Belyi maps, observe 
homotopic action of the braid group, and count possible "deformation" drawings with the given branching type.

\section{Application to algebraic Painlevé Vi functions}

As noticed in 29], 30] and [18, 1], certain Belyi coverings occur with algebraic transformations of Gauss hypergeometric solutions. These transformations are induced by pull-back transformations of a hypergeometric differential equation to a hypergeometric equation again. In particular, the Belyi covering (5.9) transforms between standard hypergeometric equations with the icosahedral and tetrahedral monodromy groups. Here is an induced hypergeometric identity:

$$
{ }_{2} \mathrm{~F}_{1}\left(\begin{array}{c}
1 / 4,-1 / 12 \\
2 / 3
\end{array} \mid x\right)=\left(1+\frac{7-33 \sqrt{-15}}{128} x\right){ }_{2}^{-1 / 12} \mathrm{~F}_{1}\left(\begin{array}{c}
11 / 60,-1 / 60 \\
2 / 3
\end{array} \mid \frac{1}{\varphi_{5}(x)}\right) .
$$

This is the same transformation as formula (50) in 29. but with a different definition of $\varphi_{5}(x)$. This formula can be checked by comparing the Taylor expansions of both sides around $x=0$.

Similarly [18, 10, almost Belyi coverings with certain ramification patterns can be used to pull back hypergeometric differential equations to $2 \times 2$ isomonodromic Fuchsian systems with four singularities. Correspondingly, one may derive algebraic solutions $y(T)$ of the sixth Painlevé equation:

$$
\begin{aligned}
\frac{d^{2} y}{d T^{2}}= & \frac{1}{2}\left(\frac{1}{y}+\frac{1}{y-1}+\frac{1}{y-T}\right)\left(\frac{d y}{d T}\right)^{2}-\left(\frac{1}{T}+\frac{1}{T-1}+\frac{1}{y-T}\right) \frac{d y}{d T} \\
& +\frac{y(y-1)(y-T)}{T^{2}(T-1)^{2}}\left(\alpha+\beta \frac{T}{y^{2}}+\gamma \frac{T-1}{(y-1)^{2}}+\delta \frac{T(T-1)}{(y-T)^{2}}\right)
\end{aligned}
$$

where $\alpha, \beta, \gamma, \delta \in \mathbb{C}$ are parameters. The standard correspondence between solutions of the sixth Painlevé equation and the mentioned isomonodromic Fuchsian systems is due to Jimbo and Miwa [16. If the singular points of the Fuchsian system are $x=0, x=1, x=T, x=\infty$, and the local monodromy differences at them are, respectively, $\theta_{0}, \theta_{1}, \theta_{T}, \theta_{\infty}$, then the corresponding Painlevé equation has the parameters

$$
\alpha=\frac{\left(\theta_{\infty}-1\right)^{2}}{2}, \quad \beta=-\frac{\theta_{0}^{2}}{2}, \quad \gamma=\frac{\theta_{1}^{2}}{2}, \quad \delta=\frac{1-\theta_{T}^{2}}{2} .
$$

We denote the corresponding Painlevé VI equation by $P_{\mathrm{VI}}\left(\theta_{0}, \theta_{1}, \theta_{T}, \theta_{\infty} ; T\right)$.

General pull-back transformations of $2 \times 2$ Fuchsian systems $d \Psi(z) / d z=$ $M(z) \Psi(z)$ have the following form:

$$
z \mapsto R(x), \quad \Psi(z) \mapsto S(x) \Psi(R(x)),
$$

where $R(x)$ is a rational function of $x$, and $S(x)$ is a Schlesinger transformation, usually designed to remove apparent singularities. For transformations to parametric isomonodromic equations, $R(x)$ and $S(x)$ may depend algebraically on parameter(s) as well. In [17, [18, [27, these pull-back transformations are called $R S$ pullback transformations, meaning that they are compositions of a rational change of the independent variable $z \mapsto R(x)$ and the Schlesinger transformation $S(x)$. The Schlesinger transformation $S(x)$ is analogous here to the projective equivalence transformations $y(x) \rightarrow \theta(x) y(x)$ of ordinary differential equations. If $S(x)$ is the identity transformation, we have a direct pullback of a Fuchsian equation. 
If $z=R(x)$ is an almost Belyi covering with a suitable ramification pattern, one can pick up hypergeometric equations (in a correspondingly normalized matrix $2 \times 2$ form) and choose appropriate Schlesinger transformations $S(x)$ so that the pull-backed Fuchsian equation would be isomonodromic and have four singular points, and there would be a corresponding algebraic solution of the sixth Painlevé equation. These $R S$-transformations are defined in [18, [19]; their algorithmic construction is considered thoroughly in [27]. The notation for suitable classes of these $R S$-pullback transformations is

$$
R S_{4}^{2}\left(\begin{array}{c|c|c}
e_{0} & e_{1} & e_{\infty} \\
P_{0} & P_{1} & P_{\infty}
\end{array}\right) .
$$

Here the subscripts 2 and 4 indicate a second-order Fuchsian system with 4 singular points after the $R S$-pullback; $P_{0}, P_{1}, P_{\infty}$ define the ramification pattern $R_{4}\left(P_{0}\left|P_{1}\right| P_{\infty}\right)$ of the almost Belyi covering $R(x)$; and $e_{0}, e_{1}, e_{\infty}$ are the local exponent differences of the hypergeometric equation.

With the almost Belyi coverings $\varphi_{12}(x), \varphi_{11}(x), \varphi_{20}(x)$ of this paper, we can construct $R S$-transformations of the types

$$
\begin{aligned}
& R S_{4}^{2}\left(\begin{array}{c|c|c}
1 / 3 & 1 / 2 & 1 / 5 \\
3+3+3+1+1+1 & 2+2+2+2+2+2 & 5+5+2
\end{array}\right), R S_{4}^{2}\left(\begin{array}{c|c|c}
1 / 3 & 1 / 2 & 2 / 5 \\
3+3+3+1+1+1 & 2+2+2+2+2+2 & 5+5+2
\end{array}\right), \\
& R S_{4}^{2}\left(\begin{array}{c|c|c}
1 / 3 & 1 / 2 & 1 / 5 \\
3+3+3+1+1 & 2+2+2+2+2+1 & 5+5+1
\end{array}\right), R S_{4}^{2}\left(\begin{array}{c|c|c}
1 / 3 & 1 / 2 & 2 / 5 \\
3+3+3+1+1 & 2+2+2+2+2+1 & 5+5+1
\end{array}\right),
\end{aligned}
$$



and derive algebraic solutions of, respectively,

$$
\begin{array}{ll}
P_{\mathrm{VI}}(1 / 3,1 / 3,1 / 3,3 / 5 ; T), & P_{\mathrm{VI}}(1 / 3,1 / 3,1 / 3,1 / 5 ; T), \\
P_{\mathrm{VI}}(1 / 3,1 / 3,1 / 2,4 / 5 ; T), & P_{\mathrm{VI}}(1 / 3,1 / 3,1 / 2,2 / 5 ; T), \\
& P_{\mathrm{VI}}(1 / 3,1 / 3,1 / 3,1 / 3 ; T) .
\end{array}
$$

All of the $R S$-pullbacks transform hypergeometric equations with the icosahedral monodromy group to isomonodromic Fuchsian systems with four singular points and the same monodromy group. The Painlevé VI solutions are called icosahedral [5]; there are 52 types of them up to branching representation of the icosahedral monodromy group, or Okamoto transformations. The solutions of (6.6) - 6.8 ) have the following Boalch types, respectively: 38, 37, 42, 43, 41. As mentioned in a footnote to the introductory section, our computations of these solutions by the method of $R S$-transformations are independent from [5].

Direct results relating $R S$-pullback transformations to algebraic Painlevé VI solutions are presented in [27. The most convenient results are reproduced here.

Theorem 6.1. Let $k_{0}, k_{1}, k_{\infty}$ denote three integers, all $\geq 2$. Let $\varphi: \mathbb{P}_{x}^{1} \rightarrow \mathbb{P}_{z}^{1}$ denote an almost Belyi map, dependent on a parameter $T$. Suppose that the following conditions are satisfied:

(i) The covering $z=\varphi(x)$ is ramified above the points $z=0, z=1, z=\infty$; there is one simply ramified point $x=y$ above $\mathbb{P}_{z}^{1} \backslash\{0,1, \infty\}$; and there are no other ramified points.

(ii) The points $x=0, x=1, x=\infty, x=T$ lie above the set $\{0,1, \infty\} \subset \mathbb{P}_{z}^{1}$. 
(iii) The points in $\varphi^{-1}(0) \backslash\{0,1, T, \infty\}$ are all ramified with the order $k_{0}$. The points in $\varphi^{-1}(1) \backslash\{0,1, T, \infty\}$ are all ramified with the order $k_{1}$. The points in $\varphi^{-1}(\infty) \backslash\{0,1, T, \infty\}$ are all ramified with the order $k_{\infty}$.

Let $a_{0}, a_{1}, a_{T}, a_{\infty}$ denote the ramification orders at $x=0,1, T, \infty$, respectively. Then the point $x=y$, as a function of $x=T$, is an algebraic solution of

$$
P_{\mathrm{VI}}\left(\frac{a_{0}}{k_{\varphi(0)}}, \frac{a_{1}}{k_{\varphi(1)}}, \frac{a_{T}}{k_{\varphi(T)}}, 1-\frac{a_{\infty}}{k_{\varphi(\infty)}} ; T\right) .
$$

Proof. This is Theorem 3.1 in [27].

Theorem 6.2. Let $z=\varphi(x)$ denote a rational covering, and let $F(x), G(x), H(x)$ denote polynomials in $x$. Let $k$ denote the order of the pole of $\varphi(x)$ at $x=\infty$. Suppose that the direct pullback of $E\left(e_{0}, e_{1}, 0, e_{\infty} ; T ; z\right)$ with respect to $\varphi(x)$ is a Fuchsian equation with the following singularities:

- Four singularities are $x=0, x=1, x=\infty$ and $x=T$, with the local monodromy differences $d_{0}, d_{1}, d_{T}, d_{\infty}$, respectively. The point $x=\infty$ lies above $z=\infty$.

- All other singularities in $\mathbb{P}_{x}^{1} \backslash\{0,1, T, \infty\}$ are apparent singularities. The apparent singularities above $z=0$ (respectively, above $z=1, z=\infty$ ) are the roots of $F(x)=0$ (respectively, of $G(x)=0, H(x)=0$ ). Their local monodromy differences are equal to the multiplicities of those roots.

Let us denote $\Delta=\operatorname{deg} F+\operatorname{deg} G+\operatorname{deg} H$, and let $\delta \leq \max (2, k)$ denote a nonnegative integer such that $\Delta+\delta$ is even. Suppose that $\left(U_{2}, V_{2}, W_{2}\right)$ is a syzygy between the three polynomials $F, G, H$, satisfying, if $\delta=0$,

(6.10) $\operatorname{deg} U_{2}=\frac{\Delta}{2}-\operatorname{deg} F, \quad \operatorname{deg} V_{2}=\frac{\Delta}{2}-\operatorname{deg} G, \quad \operatorname{deg} W_{2}<\frac{\Delta}{2}-\operatorname{deg} H$, or, if $\delta>0$,

(6.11) $\operatorname{deg} U_{2}<\frac{\Delta+\delta}{2}-\operatorname{deg} F, \quad \operatorname{deg} V_{2}<\frac{\Delta+\delta}{2}-\operatorname{deg} G, \quad \operatorname{deg} W_{2}=\frac{\Delta-\delta}{2}-\operatorname{deg} H$.

Then the numerator of the (simplified) rational function

$$
\begin{aligned}
& \frac{U_{2} W_{2}}{G}\left(\frac{\left(e_{0}-e_{1}+e_{\infty}\right)}{2} \frac{\varphi^{\prime}}{\varphi}-\frac{\left(F U_{2}\right)^{\prime}}{F U_{2}}+\frac{\left(H W_{2}\right)^{\prime}}{H W_{2}}\right) \\
& +\frac{\left(e_{0}-e_{1}-e_{\infty}\right)}{2} \frac{V_{2} W_{2}}{F} \frac{\varphi^{\prime}}{\varphi-1}+\frac{\left(e_{0}+e_{1}-e_{\infty}\right)}{2} \frac{U_{2} V_{2}}{H} \frac{\varphi^{\prime}}{\varphi(\varphi-1)}
\end{aligned}
$$

has degree 1 in $x$, and the $x$-root of the numerator is an algebraic solution of $P_{\mathrm{VI}}\left(d_{0}, d_{1}, d_{t}, d_{\infty}+\delta ; T\right)$.

Proof. This is Theorem 5.1 in [28].

The first theorem here is actually a special case of the second one, when the syzygy $\left(U_{2}, V_{2}, W_{2}\right)$ has one of the components equal to zero. The Painlevé VI solutions obtained in this case can be seen as inverse functions of particular projections [8, Figure 5] of the respective Hurwitz spaces to $\mathbb{P}_{t}^{1}$. The implied $R S$-transformation is $R S_{4}^{2}\left(\begin{array}{c|c|c}1 / k_{0} & 1 / k_{1} & 1 / k_{\infty} \\ P_{0} & P_{1} & P_{\infty}\end{array}\right)$, where $P_{0}, P_{1}, P_{\infty}$ define the ramification pattern of $\varphi(x)$. The Painlevé VI solutions can be derived without computing the Schlesinger part $S(x)$ of the $R S$-transformation. It is these solutions that are implied or computed in [10] and [18]. 
The more general Theorem 6.2 means that the same almost Belyi covering can be used to pull back several hypergeometric equations, and hence derive several algebraic Painlevé VI equations. For each implied $R S$-transformation, the Schlesinger transformations depend actually on two syzygies for the same polynomial triple $(F, G, H)$, but a single Painlevé solution depends on one syzygy, as stated in the theorem. The other syzygy determines an algebraic solution of the other (contiguous) Painlevé VI equation. When $d>\max (2, k)$, formula (6.12) still holds for a suitable syzygy $\left(U_{2}, V_{2}, W_{2}\right)$, but the syzygy is not determined by degree bounds alone; knowledge of initial terms of local solutions at $z=\infty$ of the hypergeometric equation is needed.

Both theorems have to be applied to fractional-linear normalizations of the coverings $\varphi_{12}(x), \varphi_{11}(x), \varphi_{20}(x)$, where three (of the four) singular points of the transformed Fuchsian equation are chosen to be $x=0, x=1, x=\infty$. Theorem 6.1 eventually gives solutions of $P_{\mathrm{VI}}(1 / 3,1 / 3,1 / 3,3 / 5 ; T), P_{\mathrm{VI}}(1 / 3,1 / 3,1 / 2,4 / 5 ; T)$, $P_{\mathrm{VI}}(1 / 3,1 / 3,1 / 3,1 / 3 ; T)$. These are icosahedral solutions of Boalch types 38,42 , 41 , respectively.

In particular, an $R S$-pullback $R S_{4}^{2}\left(\begin{array}{c|c|c}1 / 3 & 1 / 2 & 1 / 5 \\ 3+3+3+1+1+1 & 2+2+2+2+2+2 & 5+5+2\end{array}\right)$ with respect to $z=\varphi_{12}(x)$ is a Fuchsian system with singularities at $x=\infty$ and the roots of $G_{12}(x)=0$. The local monodromy differences are, respectively, $3 / 5,1 / 3,1 / 3,1 / 3$. A suitable normalizing fractional-linear transformation may leave $x=\infty$ invariant, but it must move two roots of $G_{12}(x)$ to the locations $x=0$ and $x=1$. Finding one root of $G_{12}$ is equivalent to considering $G_{12}(x, t)=0$ as an equation for an algebraic curve. The curve has genus 0 ; it can be parameterized as follows:

$$
t=-\frac{3(2 s+1)}{2\left(s^{3}+3 s+1\right)}, \quad x=-\frac{\left(8 s^{2}-2 s+17\right)\left(2 s^{2}+2 s+3\right)(s+1)^{2}}{10\left(s^{3}+3 s+1\right)\left(2 s^{2}+s+2\right)} .
$$

After the reparameterization of $t$ by $s$, the other two roots of $G_{12}(x)$ are equal to

$$
x=\frac{(s-2)(4 s+1)\left(32 s^{6}-8 s^{5}+164 s^{4}-94 s^{3}+91 s^{2}+2 s+18\right)}{20\left(s^{3}+3 s+1\right)\left(4 s^{2}-s+1\right)^{2}} \pm \frac{(s-2)^{2}(4 s+1)^{2}\left(8 s^{3}+6 s-1\right) w}{20(2 s+1)\left(s^{3}+3 s+1\right)\left(4 s^{2}-s+1\right)^{2}},
$$

where $w=\sqrt{(s-2)(2 s+1)\left(2 s^{2}+s+2\right)}$. It appears that the polynomial $G_{12}(x)$ splits over the function field of a genus 1 curve. Let us denote the $x$-root in (6.13) by $c_{0}$, and the latter 2 roots by $c_{+}, c_{-}$. A normalizing projective coordinate for $\mathbb{P}_{x}^{1}$ is:

$$
\lambda_{12}(x)=\frac{x-c_{+}}{c_{-}-c_{+}}
$$

Equivalently, the normalizing fractional-linear substitution to the new coordinate is given by $\lambda_{12}^{-1}(x): x \mapsto c_{+}(1-x)+c_{-} x$. By Theorem 6.1 applied to $\varphi_{12}\left(\lambda_{12}^{-1}(x)\right)$, an algebraic solution $y_{38}\left(T_{38}\right)$ of $P_{\mathrm{VI}}\left(1 / 3,1 / 3,1 / 3,3 / 5 ; T_{38}\right)$ is given by

$$
T_{38}=\lambda_{12}\left(c_{0}\right), \quad y_{38}=\lambda_{12}(0) .
$$

Explicitly, we have

$$
\begin{aligned}
& T_{38}=\frac{1}{2}+\frac{3(2 s+1)\left(32 s^{7}+32 s^{6}+138 s^{5}+25 s^{4}+130 s^{3}+30 s^{2}+20 s-10\right)}{2(s-2)^{2}(4 s+1)^{2}\left(2 s^{2}+s+2\right) \sqrt{(s-2)(2 s+1)\left(2 s^{2}+s+2\right)}}, \\
& y_{38}=\frac{1}{2}+\frac{(2 s+1)\left(32 s^{6}-8 s^{5}+164 s^{4}-94 s^{3}+91 s^{2}+2 s+18\right)}{2(s-2)(4 s+1)\left(8 s^{3}+6 s-1\right) \sqrt{(s-2)(2 s+1)\left(2 s^{2}+s+2\right)}} .
\end{aligned}
$$


To get the parameterization obtained in [5], one has to change $s \rightarrow-(s+1) / 2 s$ and the branch of the square root.

Similarly, to apply Theorem 6.1 to the degree 11 covering $\varphi_{11}(x)$, we have to compose it with a fractional-linear transformation of $\mathbb{P}_{x}^{1}$ which leaves $x=\infty$ invariant and moves the roots of $G_{11}(x)$ from (3.10) to the locations $x=0$ and $x=1$. The roots of $G_{11}(x)$ are:

$$
\begin{aligned}
x= & -\frac{27 t^{10}+270 t^{9}+945 t^{8}+2160 t^{7}+2745 t^{6}+1926 t^{5}-5 t^{4}-1340 t^{3}-1440 t^{2}-720 t-216}{2(3 t+1)^{2}\left(3 t^{2}+2 t+2\right)^{2}\left(2 t^{2}+3 t+3\right)} \\
& \pm \frac{3(t+2)^{2}\left(t^{2}+1\right)^{2}\left(3 t^{2}+2 t+2\right) \sqrt{3(t+2)(t+7)\left(3 t^{2}+3 t+2\right)}}{2(3 t+1)^{2}\left(3 t^{2}+2 t+2\right)^{2}\left(2 t^{2}+3 t+3\right)} .
\end{aligned}
$$

Let us denote these two roots by $c_{+}$and $c_{-}$. Then we can use the same expression (6.14) for the normalizing fractional-linear transformation. To distinguish, we denote this fractional-linear transformation by $\lambda_{11}(x)$. Now, let $y_{0}$ denote the extra ramification point of $\varphi_{11}$, outside the fiber of $\{0,1, \infty\} \subset \mathbb{P}_{z}^{1}$. We have:

$$
y_{0}=\frac{3 t^{5}+15 t^{4}+15 t^{3}+45 t^{2}+40 t+26}{\left(3 t^{2}+2 t+2\right)\left(5 t^{2}+4 t+3\right)} .
$$

By Theorem 6.1 applied to $\varphi_{11}\left(\lambda_{11}^{-1}(x)\right)$, an algebraic solution $y_{42}\left(T_{42}\right)$ of $P_{\mathrm{VI}}\left(1 / 3,1 / 3,1 / 2,4 / 5 ; T_{42}\right)$ is given by

$$
T_{42}=\lambda_{11}(0), \quad y_{42}=\lambda_{11}\left(y_{0}\right) .
$$

We arrive at the following Painlevé VI solution:

$$
\begin{aligned}
& T_{42}=\frac{1}{2}+\frac{27 t^{10}+270 t^{9}+945 t^{8}+2160 t^{7}+2745 t^{6}+1926 t^{5}-5 t^{4}-1340 t^{3}-1440 t^{2}-720 t-216}{6(t+2)^{2}\left(t^{2}+1\right)^{2}\left(3 t^{2}+3 t+2\right) \sqrt{3(t+2)(t+7)\left(3 t^{2}+3 t+2\right)}}, \\
& y_{42}=\frac{1}{2}+\frac{(t+7)\left(45 t^{6}+144 t^{5}+258 t^{4}+228 t^{3}+121 t^{2}+24 t-12\right)}{6\left(5 t^{2}+4 t+3\right)\left(t^{2}+1\right)(t+2) \sqrt{3(t+2)(t+7)\left(3 t^{2}+3 t+2\right)}} .
\end{aligned}
$$

The solution has genus 1 as well. To get the parameterization of the same solution obtained in [5], one may substitute $t \mapsto-(2 s-1) /(s+2)$.

To get a solution of $P_{\mathrm{VI}}(1 / 3,1 / 3,1 / 3,1 / 3 ; T)$ by Theorem 6.1, the covering $\varphi_{20}(x)$ can be composed with a fractional-linear transformation of $\mathbb{P}_{x}^{1}$ which leaves $x=\infty$ invariant and moves two roots of $H_{20}(x)$ to the locations $x=0$ and $x=1$. As in the case with $\varphi_{12}(x)$, one root of $H_{20}(x)$ can be made explicit by parameterizing the curve $H_{20}(x, t)=0$ :

$$
t=-\frac{2\left(2 s^{3}+4 s^{2}-4 s+3\right)}{5(2 s-1)^{2}}, \quad x=-\frac{16(s-2)^{2}\left(2 s^{2}+s+2\right)^{2}\left(s^{2}-2 s+6\right)}{75(2 s-1)^{4}} .
$$

After the reparameterization of $t$ by $s$, the other two roots of $H_{20}(x)$ are equal to

$$
\begin{aligned}
x= & -\frac{16 s\left(8 s^{2}-11 s+8\right)\left(56 s^{7}-166 s^{6}+318 s^{5}-269 s^{4}+31 s^{3}+75 s^{2}-28 s+8\right)}{25(2 s-1)^{8}} \\
& \pm \frac{144 s(s-1)\left(8 s^{2}-11 s+8\right)\left(2 s^{3}+4 s^{2}-4 s+3\right) \sqrt{s\left(8 s^{2}-11 s+8\right)}}{25(2 s-1)^{8}} .
\end{aligned}
$$

Let us denote the $x$-root in $(6.16)$ by $c_{0}$, and the latter 2 roots by $c_{+}, c_{-}$. Then a suitable projective parameter $\lambda_{20}(x)$ is given by the same expression as on the righthand side of (6.14). An algebraic solution $y_{41}\left(T_{41}\right)$ of $P_{\mathrm{VI}}\left(1 / 3,1 / 3,1 / 3,1 / 3 ; T_{41}\right)$ is given by

$$
T_{41}=\lambda_{20}\left(c_{0}\right), \quad y_{41}=\lambda_{20}(0) .
$$


Explicitly, we have

$$
\begin{aligned}
& T_{41}=\frac{1}{2}+\frac{(s+1)\left(32 s^{8}-320 s^{7}+1112 s^{6}-2420 s^{5}+3167 s^{4}-2420 s^{3}+1112 s^{2}-320 s+32\right)}{54 s(s-1)\left(\sqrt{s\left(8 s^{2}-11 s+8\right)}\right)^{3}} \\
& y_{41}=\frac{1}{2}-\frac{56 s^{7}-166 s^{6}+318 s^{5}-269 s^{4}+31 s^{3}+75 s^{2}-28 s+8}{18 s(s-1)\left(2 s^{3}+4 s^{2}-4 s+3\right) \sqrt{s\left(8 s^{2}-11 s+8\right)}} .
\end{aligned}
$$

To get the parameterization presented in [5, Theorem C], one has to change $s \rightarrow 1 / s$ and the branch of the square root. This solution is related via an Okamoto transformation to the Great Dodecahedron Solution [11, pages 134-143]; the DubrovinMazzocco solution solves $P_{\mathrm{VI}}\left(0,0,0,-2 / 3 ; T_{41}\right)$.

The two composite coverings $\varphi_{4} \circ \varphi_{5}$ of degree 20, with the same ramification pattern as $\varphi_{20}$, generate the same algebraic Painlevé VI solution as the degree 4 covering $\varphi_{4}$ defined in (5.10). That algebraic solution of the same equation $P_{\mathrm{VI}}\left(1 / 3,1 / 3,1 / 3,1 / 3 ; T_{41}\right)$ is parameterized in [18, Section 3.2$]$, basically using Theorem 6.1. Via the same Okamoto transformation, we get the Cube solution in [1] of $P_{\mathrm{VI}}\left(0,0,0,-2 / 3 ; T_{41}\right)$ as well. Composition of an almost Belyi covering with a Belyi covering never changes the algebraic Painlevé VI solution. Complementing computations in [18, Sections 3.1, 3.4] up to Okamoto transformations, we have all 5 Dubrovin-Mazzocco solutions in [1] now derived via the method of $R S$-pullback transformations.

Theorem 6.2 is needed to get solutions of the equations $P_{\mathrm{VI}}(1 / 3,1 / 3,1 / 3,1 / 5 ; T)$ and $P_{\mathrm{VI}}(1 / 3,1 / 3,1 / 2,2 / 5 ; T)$ of Boalch types 37 and 43 , respectively. The implied $R S$-transformations are, respectively, $R S_{4}^{2}\left(\begin{array}{c|c|c}1 / 3 & 1 / 2 & 2 / 5 \\ 3+3+3+1+1+1 & 2+2+2+2+2+2 & 5+5+2\end{array}\right)$ and $R S_{4}^{2}\left(\begin{array}{c|c|c}1 / 3 & 1 / 2 & 2 / 5 \\ 3+3+3+1+1 & 2+2+2+2+2+1 & 5+5+1\end{array}\right)$. At the end, the same normalized coverings $\varphi_{12}\left(\lambda_{12}^{-1}(x)\right)$ and $\varphi_{11}\left(\lambda_{11}^{-1}(x)\right)$ can be used. But for intermediate computations of syzygies and applications of formula (6.12), we may work with the simpler parameterized coverings $\varphi_{12}(x)$ and $\varphi_{11}(x)$. One convenient circumstance is that the point $x=\infty$ does not have to be moved.

In particular, the direct pullback of a hypergeometric equation with the local exponent differences $1 / 3,1 / 2,2 / 5$ with respect to the covering $z=\varphi_{12}(x)$ is a Fuchsian system with actual singularities at $x=\infty$ and the roots of $G_{12}(x)$, and apparent singularities at the roots of $F_{12}, P_{12}$ and $H_{12}$. The local monodromy differences at the actual singularities are $4 / 5$ or $1 / 3$, while those differences at the apparent singularities are equal to 1 or (at the roots of $H_{12}$ ) to 2 . To get rid of apparent singularities after the (implied) Schlesinger transformation $S(x)$, we have to compute syzygies between the polynomials $F_{12}, P_{12}, H_{12}^{2}$. To compute just a solution of $P_{\mathrm{VI}}(1 / 3,1 / 3,1 / 3,1 / 5 ; T)$, we apply Theorem 6.2 with $\delta=1, \Delta=12$. The suitable syzygy is unique up to constant (in $x$ ) multiples:

$$
\left(t L_{4}, 1,-4(3 t+4)^{2}\left(2 t x^{2}-t(t-8) x-(5 t+16)\left(2 t^{2}+3 t+2\right)\right)\right)
$$

where

$$
\begin{aligned}
L_{4}= & 2 t\left(112 t^{2}+307 t+208\right) x^{3}-2 t\left(-1421 t+60 t^{3}-392 t^{2}-1040\right) x^{2} \\
& -2(5 t+16)\left(90 t^{4}+251 t^{3}+183 t^{2}+27 t+16\right) x \\
& -(2 t+1)(5 t+16)^{2}\left(10 t^{3}+29 t^{2}+22 t+2\right) .
\end{aligned}
$$


The $x$-root of expression (6.12) becomes

$$
x_{37}=-\frac{(2 t+1)(5 t+4)(5 t+16)}{2\left(11 t^{2}-4 t-16\right)} .
$$

After normalization by $\lambda_{12}^{-1}$ we conclude that a solution of $P_{\mathrm{VI}}\left(1 / 3,1 / 3,1 / 3,1 / 5 ; T_{37}\right)$ is parameterized by $T_{37}=\lambda_{12}\left(c_{0}\right)$ and $y_{37}=\lambda_{12}\left(x_{37}\right)$, like the solution in (6.14). We have the solution $y_{37}\left(T_{37}\right)$ with $T_{37}=T_{38}$ and

$$
y_{37}=\frac{1}{2}-\frac{(2 s+1)\left(256 s^{8}-832 s^{7}-800 s^{6}-3232 s^{5}-1844 s^{4}-2950 s^{3}-1436 s^{2}-391 s+64\right)}{2(4 s+1)\left(64 s^{6}+336 s^{4}+104 s^{3}+36 s^{2}-132 s-59\right) \sqrt{(s-2)(2 s+1)\left(2 s^{2}+s+2\right)}} .
$$

To get the parameterization obtained in [5], one may substitute $s \rightarrow-(s+1) /(2 s)$.

Similarly, the direct pullback of the same hypergeometric equation with respect to the covering $z=\varphi_{11}(x)$ has apparent singularities at the roots of $F_{11}, P_{11}$ and $H_{11}$. For a solution of $P_{\mathrm{VI}}(1 / 3,1 / 3,1 / 2,2 / 5 ; T)$, we have to compute syzygies between the polynomials $F_{11}, P_{11}, H_{11}^{2}$, assuming $\delta=0, \Delta=10$. A suitable syzygy is:

$$
\left(L_{5}, x-(3 t+1)^{2},-30 \frac{3 t^{2}+3 t+2}{3 t+1}\left(\left(18 t^{2}+13 t+9\right) x-(t-1)(3 t+1)^{3}\right)\right),
$$

where $L_{5}$ can be computed easily knowing the other two components. The $x$-root of expression (6.12) becomes

$$
x_{43}=\frac{(3 t+1)^{2}\left(13 t^{5}+65 t^{4}+165 t^{3}+195 t^{2}+140 t+46\right)}{3\left(3 t^{2}+2 t+2\right)\left(5 t^{6}+30 t^{5}+45 t^{4}+22 t^{3}-13 t^{2}-16 t-9\right)} .
$$

After normalization by $\lambda_{11}^{-1}$ we conclude that a solution of $P_{\mathrm{VI}}(1 / 3,1 / 3,1 / 2,2 / 5$; $\left.T_{43}\right)$ is parameterized by $T_{43}=\lambda_{11}(0), y_{43}=\lambda_{11}\left(x_{43}\right)$. We have the solution $y_{43}\left(T_{43}\right)$ with $T_{43}=T_{42}$ and

$$
y_{43}=\frac{1}{2}+\frac{(t+7)\left(135 t^{9}+540 t^{8}+1530 t^{7}+2916 t^{6}+3714 t^{5}+3486 t^{4}+2278 t^{3}+1144 t^{2}+399 t+114\right)}{18\left(t^{2}+1\right)\left(5 t^{6}+30 t^{5}+45 t^{4}+22 t^{3}-13 t^{2}-16 t-9\right) \sqrt{3(t+2)(t+7)\left(3 t^{2}+3 t+2\right)}} .
$$

To get the parameterization obtained in [5, one may substitute $t \rightarrow-(2 s-1) /(s+2)$.

\section{REFERENCES}

1. F. V. Andreev and A. V. Kitaev, Some examples of $R S_{3}^{2}(3)$-transformations of ranks 5 and 6 as the higher order transformations for the hypergeometric function, Ramanujan J., 7 , no. 4 (2003), pp. 455-476. MR2040984 (2004k:33005)

2. F. V. Andreev and A. V. Kitaev, Transformations $R S_{4}^{2}(3)$ of the ranks $\leq 4$ and algebraic solutions of the sixth Painlevé equation, Comm. Math. Phys. 228 (2002), pp. 151-176. MR.1911252 (2003f:34186)

3. E. A. Arnold, Modular algorithms for computing Gröbner bases, Journal of Symbolic Computation, 35, no. 4 (2003), pp. 403-419. MR1976575 (2004c:13044)

4. G. V. Belyi, Galois extensions of a maximal cyclotomic field (Russian), Izv. Akad. Nauk SSSR Ser. Mat. 43, no. 2, (1979), pp. 267-276. English Translation in Math. USSR Izv. 14 (1980), pp. 247-256. MR534593 (80f:12008)

5. P. Boalch, The fifty-two icosahedral solutions to Painlevé. VI, J. Reine Angew. Math. 596 (2006), pp. 183-214. MR2254812(2007i:34149)

6. P. Boalch, Some explicit solutions to the Riemann-Hilbert problem, in "Differential Equations and Quantum Groups", IRMA Lectures in Mathematics and Theoretical Physics, Vol. 9 (2006), pp. 85-112. MR2322328 (2008h:34145)

7. P. Boalch, Higher genus icosahedral Painlevé curves, Funk. Ekvac. 50 (2007), pp. 19-32. MR:2332077 (2008c:34188) 
8. J.-M. Couveignes, Tools for the computation of families of coverings, In "Aspects of Galois theory", London Math. Soc. Lecture Notes Ser., Vol. 256, Cambridge Univ. Press, 1999, pp. 38-65. MR1708601 (2000f:14037)

9. S. Diaz, R. Donagi, and D. Harbater, Every curve is a Hurwitz space, Duke Math. J., 59, no. 3 (1989), pp. 737-746. MR.1046746 (91i:14021)

10. Ch. F. Doran, Algebraic and geometric isomonodromic deformations, J. Differential Geometry 59 (2001), pp. 33-85. MR.1909248 (2004d:32013)

11. B. Dubrovin and M. Mazzocco, Monodromy of certain Painlevé-VI transcendents and reflection groups, Invent. Math. 141 (2000), pp. 55-147. MR1767271 (2001j:34114)

12. A. Grothendieck, Esquisse d'un programme, In Schneps L., Lochak P. (Eds.), "Geometric Galois Actions I", London Math. Soc. Lecture Note Ser., Vol. 242, Cambridge Univ. Press, 1997, pp. 5-48. English translation: the same volume, pp. 243-284. MR1483107 (99c:14034)

13. G.-M. Greuel, G. Pfister, H. Schönemann, Singular 2.0.3. A Computer Algebra System for Polynomial Computations, Centre for Computer Algebra, University of Kaiserslautern (2005). http://www.singular.uni-kl.de.

14. R. Hartshorne, Algebraic Geometry, Springer-Verlag, New York, 1977. MR0463157 (57:3116)

15. J. A. Hempel, Existence conditions for a class of modular subgroups of genus zero, Bull. Austr. Math. Soc. 66 (2002), pp. 517-525. MR1939212 (2003j:20085)

16. M. Jimbo and T. Miwa, Monodromy preserving deformation of linear ordinary differential equations with rational coefficients. II, Physica 2D (1981), pp. 407-448. MR625446 (83k:34010b)

17. A. V. Kitaev, Special functions of isomonodromy type, rational transformations of the spectral parameter, and algebraic solutions of the Sixth Painlevé Equation (Russian), Algebra i Analiz 14, no. 3, (2002) pp. 121-139. English Translation in St. Petersburg Math. J. 14, no. 3, (2003) pp. 453-465. MR.1921990 (2003e:34168)

18. A. V. Kitaev, Grothendieck's Dessins d'Enfants, Their Deformations and Algebraic Solutions of the Sixth Painlevé and Gauss Hypergeometric Equations, Algebra i Analiz 17, no. 1 (2005), pp. 224-273. MR.2140681 (2006b:33040)

19. A. V. Kitaev, Remarks Towards Classification of $R S_{4}^{2}(3)$-Transformations and Algebraic Solutions of the Sixth Painlevé Equation, Proceedings of the Angers Conference "Asymptotic Theories and Painlevé Equations" (June 01-05, 2004). Sèminaires et Congrès 14 (2006), pp. 199-227. See: smf.emath.fr/en/Publications/SeminairesCongres/2006/14/html. MR 2353466

20. E. Kreines, On families of geometric parasitic solutions for Belyi systems of genus zero, Fundamentalnaya i Priklandaya Matematika 9 (2003), pp. 103-111. Available at http://ellib.itep.ru/mathphys/psfiles/02_48.ps MR2072622(2005e:14038)

21. A. K. Lenstra, H. W. Lenstra, Jr., and L. Lovasz, Factoring polynomials with rational coefficients, Math. Ann. 261 (1982), pp. 515-534. MR682664 (84a:12002)

22. K. Magaard, S. Shpectorov and H. Völklein, A GAP package for braid orbit computation and applications, Experimental Mathematics, 12 (2003), no. 4, pp. 385-393. MR.2043989 (2005e:12007)

23. Mohamed El Marraki, Nicolas Hanusse, Jorg Zipperer, and Alexander Zvonkin, Cacti, braids and complex polynomials, Séminaire Lotharingien de Combinatoire 37 (1996). Available at http://citeseer.ist.psu.edu/259789.html. MR.1462334(98j:57003)

24. G. Shabat, On a class of families of Belyi functions, in "Formal Power Series and Algebraic Combinatorics", D. Krob, A. A. Mikhalev, A. V. Mikhalev (Eds.), Springer-Verlag, Berlin, Heidelberg, 2000; pp. 575-581. MR 1798251 (2001m:30007)

25. L. Schneps, Dessins d'enfant on the Riemann sphere, In "The Grothendieck theory of Dessins d'Enfant", London Math. Soc. Lecture Notes Ser., Vol. 200, Cambridge Univ. Press, 1994; pp. 38-65. MR1305393 (95j:11061)

26. R. Vidūnas and A. V. Kitaev, Quadratic transformations of the sixth Painlevé equation with application to algebraic solutions, Mathematische Nachrichten 280 (2007), pp. 1834-1855. MR 2365021

27. R. Vidūnas and A. V. Kitaev, Computation of RS-pullback transformations for algebraic Painlevé VI solutions. Available at http://arxiv.org/abs/0705.2963.

28. R. Vidūnas and A. V. Kitaev, Schlesinger transformations for algebraic Painlevé VI solutions. Available at http://arxiv.org/abs/0810.2766. 
29. R. Vidūnas, Algebraic Transformations of Gauss Hypergeometric Functions, Accepted by Funk. Ekvac. Available at http://www.arxiv.org/math.CA/0408269 (2004).

30. R. Vidūnas, Transformations of some Gauss hypergeometric functions, J. Comp. Appl. Math. 178 (2005), pp. 473-487. MR2127899 (2006a:33003)

31. P. S. Wang, M. J. T. Guy and J. H. Davenport, P-adic reconstruction of rational numbers, SIGSAM Bulletin, Vol. 16, ACM, 1982, pp. 2-3.

32. L. Zapponi, Galois action on diameter four trees, preprint http://www.arxiv.org/math.AG/ 0108031 (2001).

33. A. Zvonkin, Megamaps: Construction and Examples, Discrete Mathematics and Theoretical Computer Science Proceedings AA (DM-CCG), 2001, pp. 329-340. MR.1888783|(2003d:14036)

Department of Mathematics, Kyushu University, Fukuoka 812-8581, Japan

Current address: Department of Mathematics, Kobe University, Rokko-dai 1-1, Nada-ku, Kobe 657-8501, Japan

E-mail address: rvidunas@gmail.com

School of Mathematics and Statistics, University of Sydney, NSW 2006, Australia

Current address: Steklov Mathematical Institute, Fontanka 27, St. Petersburg 191023, Russia

E-mail address: kitaev@pdmi.ras.ru 